\section{Associação entre excesso de peso e obesidade e mortalidade em capitais brasileiras e províncias argentinas}

\author{
Association of excess weight and obesity and
mortality in Brazilian state capitals and \\ Association of excess weight and obesity and
mortality in Brazilian state capitals and \\ Argentine provinces
}

\author{
Asociación entre exceso de peso, obesidad y \\ mortalidad en capitales brasileñas y \\ provincias argentinas
}

Valeria Romina Amann 1

Leonardo Pozza dos Santos 2

Denise Petrucci Gigante 1,3

\section{Resumo}

O objetivo deste trabalho foi avaliar a associação da prevalência de excesso de peso e obesidade com as taxas de mortalidade total e específica nas capitais brasileiras e províncias argentinas. Estudo ecológico com dados secundários, em que as exposições principais foram as prevalências de excesso de peso e obesidade estimadas com base em dados da pesquisa Vigilância de Fatores de Risco e Proteção para Doenças Crônicas por Inquérito Telefônico (Vigitel) de 2014 (Brasil) e da Pesquisa Nacional de Fatores de Risco de 2013 (Argentina). As taxas de mortalidade geral e especificas para o ano de 2015 foram obtidas no Sistema de Informações sobre Mortalidade do Departamento de Informática do SUS (Brasil) e na Direção de Estatísticas e Informações de Saúde do Ministério da Saúde (Argentina). As taxas de mortalidade brasileiras foram padronizadas considerando-se a estrutura etária da população argentina como padrão. Regressão linear bruta e ajustada foi utilizada para avaliar a associação das prevalências de excesso de peso e obesidade com as taxas de mortalidade geral e específicas. Após o ajuste para os potenciais fatores de confusão, a prevalência de obesidade se associou positivamente com a taxa de mortalidade geral tanto no Brasil ( $\beta=0,18 ;$ IC95\%: 0,$01 ; 0,35)$ quanto na Argentina ( $\beta=0,06$; IC95\%: 0,01; 0,13). Para as demais taxas de mortalidade (cardiovascular e por câncer) não houve associação. Conclui-se que as capitais brasileiras e províncias argentinas com maiores prevalências de obesidade apresentam maiores taxas de mortalidade geral, sendo esta associação inconsistente para as taxas específicas.

Índice de Massa Corporal; Mortalidade; Estudo Observacional

\author{
Correspondência \\ V. R. Amann \\ Programa de Pós-graduação em Nutrição e Alimentos, \\ Universidade Federal de Pelotas. \\ Rua Gomes Carneiro 1, Pelotas, RS 96010-610, Brasil. \\ valeriaamann@hotmail.com
1 Programa de Pós-graduação em Nutrição e Alimentos, Universidade Federal de Pelotas, Pelotas, Brasil.
2 Curso de Nutrição, Universidade Federal do Pampa, Itaqui, Brasil.
3 Programa de Pós-graduação em Epidemiologia, Universidade Federal de Pelotas, Pelotas, Brasil.




\section{Introdução}

Nos últimos anos, as doenças crônicas não transmissíveis (DCNT) foram as principais causas de mortalidade no mundo, provocando em média, a cada ano, $70 \%$ das mortes, sendo mais de $40 \%$ destas mortes em pessoas com até 70 anos de idade 1. Os principais fatores de risco comportamentais para DCNT 1 são o tabagismo, o consumo excessivo de álcool, o sedentarismo e o excesso de peso - índice de massa corporal (IMC) $\geq 25 \mathrm{~kg} / \mathrm{m}^{2}$-, sendo este último um problema de saúde pública que acomete mais da metade da população adulta na maioria dos países, dentre eles o Brasil 2 e a Argentina 3.

De acordo com estimativas recentes, a prevalência de excesso de peso no Brasil é de $56,9 \%$ 2, e na Argentina chega a quase $60 \% 3$. Na realidade, o excesso de peso parece ser um problema generalizado na América Latina, visto que países como Paraguai, Peru e Chile também apresentam prevalências elevadas $4,5,6$, sendo que no Chile quase $70 \%$ dos adultos apresentam IMC $\geq 25 \mathrm{~kg} / \mathrm{m}^{2} 6$. Com relação à obesidade, em torno de $20 \%$ da população adulta brasileira e argentina apresentam IMC $\geq 30 \mathrm{~kg} / \mathrm{m}^{2}$, sendo similar à ocorrência observada em países de renda alta como a Inglaterra, onde $26 \%$ da população adulta apresentam obesidade 7 .

Em países de renda alta, a relação entre excesso de peso e mortalidade vem sendo debatida, e uma meta-análise 8 conduzida em 2013 concluiu que a obesidade está associada com o aumento do risco de morte. No entanto, esse risco só foi observado para os graus mais elevados de obesidade (IMC > $35 \mathrm{~kg} / \mathrm{m}^{2}$ ), já o sobrepeso (IMC entre 25 e $29,9 \mathrm{~kg} / \mathrm{m}^{2}$ ) foi considerado um fator de proteção para a mortalidade geral, quando comparado com o IMC normal 8. Outros estudos mais recentes também encontraram resultados semelhantes aos dessa meta-análise 9,10,11.

Em contrapartida, há evidências que apontam que o menor risco de mortalidade pode ser observado naqueles indivíduos que apresentam IMC na faixa da normalidade, isto é, entre 20 e $25 \mathrm{~kg} / \mathrm{m}^{2}$, e qualquer nível de sobrepeso e obesidade já elevariam o risco de mortalidade geral 12,13. A associação entre mortalidade com qualquer grau de sobrepeso ou obesidade também foi observada em outra meta-análise, publicada em 2016, e que incluiu 239 estudos prospectivos de quatro continentes 14 .

A maioria dos trabalhos que tem explorado a associação entre excesso de peso e mortalidade tem sido realizada em países de alta renda, sendo escassas as evidências para esta relação oriundas de estudos de base populacional tanto no Brasil quanto na Argentina. Nesse sentido, um estudo ecológico de base populacional em ambos os países é útil para mostrar como acontece essa associação no nível agregado, em dois dos países mais populosos e com maior economia da América do Sul.

Assim, em consonância com as metas dos Objetivos de Desenvolvimento Sustentável das Nações Unidas, que, entre outras coisas, propõem reduzir em um terço a mortalidade prematura por doenças não transmissíveis até 2030 15, e diante da controversa relação entre excesso de peso e mortalidade, com a ausência de trabalhos similares na população adulta do Brasil e da Argentina, o presente artigo objetivou estudar a relação entre a prevalência de excesso de peso e obesidade com as taxas de mortalidade geral e específicas nas capitais brasileiras e províncias argentinas.

\section{Métodos}

\section{Delineamento e amostra}

Estudo observacional ecológico cujas unidades de análise foram as capitais brasileiras e as províncias argentinas. Os dados do presente trabalho foram extraídos da pesquisa denominada Vigilância de Fatores de Risco e Proteção para Doenças Crônicas por Inquérito Telefônico (Vigitel) de 2014, realizada nas capitais brasileiras, e da III Pesquisa Nacional de Fatores de Risco (ENFR; III Encuesta Nacional de Factores de Riesgo) de 2013, realizada nas províncias argentinas. São dois estudos de base populacional e corte transversal, cuja população-alvo incluiu indivíduos adultos com idades iguais ou superiores a 18 anos.

O Vigitel é um inquérito telefónico de base populacional, realizado anualmente e cujo objetivo é monitorar a distribuição e frequência de fatores de risco e de proteção para DCNT, assim como descrever a evolução anual destes fatores na população adulta (18 anos ou mais) das 26 capitais brasileiras e do Distrito Federal. A amostragem do Vigitel consiste em duas etapas: a primeira refere-se a um sorteio sistemático e estratificado de cerca de 5 mil linhas telefônicas de cada cidade, pelos códigos 
de endereçamento postal (CEP), sendo que, em 2014, foram sorteadas 135 mil linhas de telefonia fixa. Na segunda etapa, um dos adultos que reside no domicílio selecionado é sorteado para responder ao questionário. Em 2014, foram realizadas 40.853 entrevistas 16.

A ENFR é um inquérito domiciliar de base populacional e de corte transversal, que tem como objetivo monitorar a evolução dos principais fatores de risco para DCNT, assim como proporcionar informação válida e confiável também sobre o processo de atenção médica e prevalência das DCNT. Trata-se de um estudo conduzido a cada quatro anos com a população adulta argentina (18 anos ou mais), residente nas 23 províncias e no Distrito Federal, sendo que na ENFR de 2013 foram realizadas 41.108 entrevistas, nas quais todos os dados, incluindo as informações antropométricas, foram autorreferidos ${ }^{17}$. A amostragem da ENFR é feita em quatro etapas, com as três primeiras incluídas na Amostra Mestre Urbana de Vivendas da República Argentina (Muestra Maestra Urbana de Viviendas de la República Argentina), que é uma amostra probabilística de áreas cujo domínio é o total de localidades com 2 mil habitantes ou mais. Na quarta etapa, selecionou-se uma pessoa com idade igual ou maior de 18 anos para responder ao questionário utilizando-se a tabela de Kish 18.

\section{Estado nutricional e características comportamentais e sociodemográficas das capitais/províncias}

Como fatores de exposição do presente estudo, as prevalências de excesso de peso e obesidade foram estimadas para as capitais brasileiras e províncias argentinas. A prevalência de excesso de peso foi definida como o total de pessoas com IMC $\geq 25 \mathrm{~kg} / \mathrm{m}^{2}$, dividido pela população total da capital/ província e multiplicado por 100. Já a prevalência de obesidade foi definida como o total de pessoas com IMC $\geq 30 \mathrm{~kg} / \mathrm{m}^{2}$, dividido pelo total da população da capital/província e multiplicado por 100 . Também foi estimada a prevalência de IMC normal, definida como o total de pessoas com IMC entre 18,5 e $24,9 \mathrm{~kg} / \mathrm{m}^{2}$, dividido pelo total da população da capital/província e multiplicado por 100 . Todas as prevalências estimadas foram ponderadas levando-se em consideração os fatores de expansão aplicados nas duas pesquisas, devido ao complexo desenho amostral do Vigitel e da ENFR.

Além das variáveis referentes ao estado nutricional da população de cada capital/província, foram obtidas informações sobre as características sociodemográficas oriundas das entrevistas realizadas em ambas as pesquisas. Assim, foram incluídas no presente trabalho a média de idade dos entrevistados e as distribuições por sexo (proporção de indivíduos do sexo masculino) e escolaridade de cada capital/província (proporção de indivíduos com, pelo menos, Ensino Médio completo).

As características comportamentais como a proporção de consumo regular de frutas e hortaliças, de inatividade física, de consumo abusivo de álcool e prevalência de tabagismo também foram incluídas na análise, uma vez que são fatores potencialmente relacionados com as exposições de interesse e os desfechos estudados. No Vigitel, o consumo de frutas e hortaliças foi considerado regular quando realizado cinco ou mais vezes por dia, em cinco ou mais dias da semana. Já na Argentina, o consumo de frutas e hortaliças foi classificado como regular quando os indivíduos relataram ingerir cinco porções de frutas e hortaliças por dia. Em ambas as pesquisas, a inatividade física foi considerada como a não realização de atividade física ou a realização de menos de 150 minutos por semana, de acordo com as recomendações mundiais de atividade física para essa idade 19. O consumo excessivo de álcool foi classificado como abusivo quando cinco ou mais doses (homens) ou quatro ou mais doses (mulheres) de bebidas alcoólicas foram consumidas em uma única ocasião, pelo menos uma vez, nos últimos 30 dias. Por fim, a prevalência de tabagismo foi calculada com base no total de indivíduos que relataram ser fumantes no momento da entrevista em ambas as pesquisas.

O Índice de Desenvolvimento Humano (IDH), que tem a finalidade de avaliar o desenvolvimento econômico dos países, foi incluído nas análises por ser potencialmente associado com as exposições e os desfechos de interesse no presente estudo. O IDH de cada capital/província foi obtido, no Brasil, pelas informações do Instituto Brasileiro de Geografia e Estatística (IBGE), para o ano de 2010 e, na Argentina, pelo Informe Nacional sobre o Desenvolvimento Humano de 2011. 
Os desfechos do presente estudo, taxas de mortalidade geral e específicas, foram calculados com base nos bancos nacionais de mortalidade para o ano de 2015 de ambos os países. No Brasil, os dados foram obtidos no Sistema de Informações sobre Mortalidade (SIM) do Departamento de Informática do SUS (DATASUS), e na Argentina estavam disponíveis na Direção de Estatísticas e Informações de Saúde (DEIS) do Ministério da Saúde daquele país. Os dados foram coletados por local de residência do óbito e segundo faixa etária.

Baseando-se nas estimativas da população de cada capital/província para o ano de 2015 do IBGE 20 e do Instituto Nacional de Estatísticas e Censos (INDEC) 21 da Argentina, foram obtidos os dados de população de cada unidade de análise, os quais foram usados para a obtenção das taxas de mortalidade geral e específica. A taxa de mortalidade geral por mil habitantes para o ano de 2015 foi calculada valendo-se da divisão entre o número total de mortes em 2015 pelo total da população deste ano a cada mil habitantes.

As taxas de mortalidade específicas foram determinadas para as mortes por cânceres dependentes de hormônios e por doenças cardiovasculares, identificadas segundo a Classificação Internacional de Doenças - 10a Revisão (CID-10). A taxa de mortalidade por cânceres dependentes de hormônios incluiu as mortes causadas pelos seguintes CIDs: C15-C26 (neoplasias malignas dos órgãos digestivos), C50 (neoplasia maligna da mama), C51-C58 (neoplasias malignas dos ovários, colo e corpo uterino), C61 (neoplasia maligna da próstata), C73 (neoplasia maligna da glândula tireoide), C90 (mieloma múltiplo) e D32 (meningioma). Já a taxa de mortalidade por doenças cardiovasculares incluiu os seguintes códigos: I10-I15 (doenças hipertensivas), I20-I25 (doenças isquêmicas do coração), I49-I50 (insuficiência cardíaca), I60-I69 (doenças cerebrovasculares), I70 (aterosclerose) e I74 (embolia e trombose arterial) 22 .

Por fim, as taxas de mortalidade específicas por cânceres dependentes de hormônios e por doenças cardiovasculares foram calculadas baseando-se no número de mortes por determinada condição no total da população a cada 100 mil habitantes.

\section{Análises estatísticas}

A correlação de Pearson foi utilizada para avaliar a associação das prevalências de excesso de peso e obesidade com as taxas de mortalidade geral e específicas das capitais brasileiras e províncias argentinas. Mesmo com uma leve assimetria na distribuição dos desfechos, optou-se por usar a correlação de Pearson com base no Teorema do Limite Central que indica que as médias amostrais de uma determinada população tendem a distribuição normal 23 .

A regressão linear foi utilizada para avaliar o efeito bruto e ajustado das variáveis independentes (estado nutricional) sobre as taxas de mortalidade. Para essa análise, as taxas de mortalidade geral e específicas das capitais brasileiras foram padronizadas, mediante método de padronização direta 24 , utilizando-se a população argentina como padrão. Assim, foi possível estimar as taxas de mortalidade geral e específicas das capitais brasileiras caso apresentassem a mesma estrutura etária e de gênero da população argentina.

No modelo de regressão linear ajustado, foram incluídas aquelas covariáveis que apresentaram associação com pelo menos uma exposição e um desfecho, em pelos menos um país, considerandose um nível de significância de $20 \%$. Optou-se por esse critério a fim de manter o modelo de ajuste similar em todas as análises, independentemente do país. Sendo assim, foram considerados como potenciais fatores de confusão as características sociodemográficas (IDH e escolaridade) e comportamentais (consumo regular de frutas e hortaliças e consumo excessivo de álcool). No modelo de ajuste para as províncias argentinas, incluiu-se também a média de idade dos entrevistados de cada província como potencial fator de confusão. Já nas análises realizadas no Brasil, optou-se por não incluir essa variável no modelo por duas razões: aumento importante na colinearidade do modelo como um todo após a inclusão da idade, e pelo fato de as taxas de mortalidade já estarem ajustadas pela estrutura etária da população argentina.

Em todo o processo de análise foi investigado o Fator de Inflação da Variância (VIF) a fim de detectar a presença de multicolinearidade nos modelos estudados. As análises estatísticas foram 
realizadas no pacote estatístico Stata, versão 12.0 (https://www.stata.com). Este estudo foi aprovado pelo Comitê de Ética em Pesquisa da Faculdade de Medicina da Universidade Federal de Pelotas, sob parecer no 2.241.678.

\section{Resultados}

\section{Caracterização da amostra}

No Vigitel de 2014, a média de idade dos indivíduos entrevistados foi de 40,5 anos, e na ENFR esta média foi de 42,1 anos, com a maioria da população entrevistada em ambos os países sendo do sexo feminino. Quanto às características socioeconômicas, o IDH médio brasileiro foi de 0,78 , variando de 0,75 nas capitais da Região Norte a 0,83 nas capitais da Região Sul. Já na Argentina, o IDH médio foi de 0,84, variando de 0,82 nas províncias do Nordeste a 0,86 nas províncias das regiões Pampeana e Patagônica. Com relação à escolaridade, a proporção média de indivíduos brasileiros com no mínimo Ensino Médio completo foi de 65,5\%, e na Argentina foi de apenas 51\% (Tabela 1).

\section{Estado nutricional no Brasil e na Argentina}

A média de IMC ficou acima de $25 \mathrm{~kg} / \mathrm{m}^{2}$ em todas as capitais brasileiras e províncias argentinas. A prevalência média de excesso de peso no Brasil foi de 52,2\% e na Argentina tal prevalência chegou a 58,7\%. Com relação à obesidade, a prevalência média nas 27 capitais brasileiras foi de $18,1 \%$ e de 21,1\% nas províncias Argentinas (as prevalências de excesso de peso e obesidade nas capitais/províncias incluídas no estudo, de acordo com as macrorregiões geográficas de cada país, são apresentadas na Tabela 1).

Analisando a associação entre as características socioeconômicas e as prevalências de excesso de peso e obesidade, observou-se que as capitais brasileiras com maior IDH apresentaram tendência de menor prevalência de obesidade (valor de $\mathrm{p}$ de tendência $=0,04$ ). Já na Argentina, o IDH não esteve associado à situação nutricional nas províncias (dados não apresentados em tabela).

\section{Mortalidade geral e específica no Brasil e na Argentina}

A taxa de mortalidade geral foi de 6,2 mortes por mil habitantes entre as capitais brasileiras e de 7,7 mortes por mil habitantes nas províncias argentinas. Em ambos os países houve diferença significativa nas taxas de mortalidade geral das capitais/províncias de acordo com as macrorregiões geográficas (valor de $\mathrm{p}=0,02$ no Brasil e < 0,001 na Argentina). Ainda na Tabela 1 é apresentada a distribuição das taxas de mortalidade por causas específicas de acordo com as macrorregiões geográficas de cada país. No Brasil, diferenças significativas por regiões foram observadas para as taxas de mortalidade por cânceres e por doenças cardiovasculares, sendo que as capitais da Região Norte apresentaram as menores taxas e as capitais das regiões Sul e Sudeste registraram taxas maiores. Na Argentina, diferenças também foram observadas, com as províncias das regiões Pampeana e Cuyo apresentando os maiores valores para ambas as taxas de mortalidade específicas, e a menor taxa de mortalidade por câncer foi observada nas províncias da Região Nordeste e a menor taxa para as doenças cardiovasculares nas províncias da Patagônica.

Ainda no Brasil, após padronização das taxas de mortalidade com base no sexo e na estrutura etária da população argentina, observou-se que houve um aumento nas taxas de mortalidade das capitais das regiões Norte, Nordeste e Centro-oeste. Além disso, a diferença nas taxas de mortalidade de acordo as regiões geográficas do país não foi mais observada após a padronização (Tabela 1). 
Tabela 1

Caracterização das variáveis sociodemográficas e do estado nutricional nos anos 2013/2014 e de mortalidade em 2015 nas capitais/províncias, de acordo com as macrorregiões do Brasil e da Argentina.

\begin{tabular}{|c|c|c|c|c|c|c|}
\hline \multirow[t]{2}{*}{ Variáveis } & \multicolumn{6}{|c|}{ Brasil (Regiões) } \\
\hline & Norte & Nordeste & Centro-oeste & Sudeste & Sul & Valor de $p$ * \\
\hline \multicolumn{7}{|l|}{ Características sociodemográficas } \\
\hline $\mathrm{IDH}$ & 0,75 & 0,76 & 0,80 & 0,82 & 0,83 & 0,000 \\
\hline Escolaridade (proporção) & 65,32 & 63,00 & 66,37 & 67,06 & 70,38 & 0,247 \\
\hline Idade média & 38,06 & 40,51 & 40,62 & 42,96 & 42,97 & 0,000 \\
\hline \multicolumn{7}{|l|}{ Estado nutricional } \\
\hline Prevalência de excesso de peso & 53,08 & 51,49 & 52,41 & 51,42 & 53,22 & 0,737 \\
\hline Prevalência de obesidade & 19,05 & 17,64 & 18,52 & 17,21 & 17,99 & 0,655 \\
\hline \multicolumn{7}{|l|}{ Taxas de mortalidade brutas } \\
\hline Mortalidade geral (média) & 4,67 & 6,07 & 5,51 & 6,56 & 6,03 & 0,020 \\
\hline Mortalidade por cânceres (média) & 42,85 & 56,18 & 58,46 & 73,08 & 81,41 & 0,000 \\
\hline Mortalidade por doenças cardiovasculares (média) & 84,96 & 127,29 & 119,51 & 144,11 & 130,75 & 0,010 \\
\hline \multicolumn{7}{|l|}{ Taxas de mortalidade padronizadas } \\
\hline Mortalidade geral (média) & 7,77 & 7,64 & 8,06 & 7,05 & 6,61 & 0,474 \\
\hline Mortalidade por cânceres (média) & 76,18 & 70,99 & 80,84 & 75,96 & 85,73 & 0,454 \\
\hline Mortalidade por doenças cardiovasculares (média) & 176,68 & 172,55 & 182,77 & 158,25 & 148,19 & 0,725 \\
\hline \multirow[t]{2}{*}{ Variáveis } & \multicolumn{6}{|c|}{ Argentina (Regiões) } \\
\hline & Noroeste & Nordeste & Cuyo & Pampeana & Patagônica & Valor de $p$ * \\
\hline \multicolumn{7}{|l|}{ Características sociodemográficas } \\
\hline IDH & 0,83 & 0,82 & 0,84 & 0,86 & 0,86 & 0,000 \\
\hline Escolaridade (proporção) & 51,45 & 45,75 & 52,13 & 54,20 & 50,18 & 0,546 \\
\hline Idade média & 41,19 & 41,14 & 42,82 & 41,00 & 44,18 & 0,004 \\
\hline \multicolumn{7}{|l|}{ Estado nutricional } \\
\hline Prevalência de excesso de peso & 60,13 & 57,21 & 57,56 & 61,52 & 56,56 & 0,180 \\
\hline Prevalência de obesidade & 22,30 & 20,02 & 19,69 & 19,67 & 23,13 & 0,171 \\
\hline \multicolumn{7}{|l|}{ Taxas de mortalidade } \\
\hline Mortalidade geral (média) & 6,14 & 6,28 & 6,87 & 8,42 & 5,05 & 0,001 \\
\hline Mortalidade por cânceres (média) & 56,69 & 63,04 & 80,24 & 95,00 & 64,14 & 0,000 \\
\hline Mortalidade por doenças cardiovasculares (média) & 108,33 & 141,57 & 176,67 & 193,42 & 94,70 & 0,000 \\
\hline
\end{tabular}

IDH: Índice de Desenvolvimento Humano.

* Análise de variância (ANOVA).

\section{Taxas de mortalidade geral e específicas segundo o estado nutricional}

Os coeficientes de correlação entre as taxas de mortalidade geral e específica e as prevalências de excesso de peso e obesidade nas capitais brasileiras e províncias argentinas são apresentados na Tabela 2. Nas capitais brasileiras, correlações fracas e não significativas foram evidenciadas entre as taxas de mortalidade geral e as prevalências do estado nutricional. Após a padronização pela distribuição de sexo e de idade da Argentina, essas correlações se acentuaram e foram positivas para as prevalências de excesso de peso $(r=0,47$; valor de $\mathrm{p}=0,014)$ e obesidade $(\mathrm{r}=0,53$; valor de $\mathrm{p}=0,004)$ e negativas para a prevalência de indivíduos classificados com estado nutricional adequado $(\mathrm{r}=-0,45$; valor de $\mathrm{p}=0,020)$ com as taxas de mortalidade geral. Na Argentina, correlações moderadas foram observadas, porém no sentido inverso do que foi encontrado no Brasil, sendo positivas entre a taxa de mortalidade geral e a prevalência de indivíduos com IMC normal $(r=0,51$; valor de $\mathrm{p}=0,011)$ e negativas entre a 
Correlação entre as prevalências do estado nutricional de 2013/2014 e taxas de mortalidade geral e específicas no ano de 2015 no Brasil e na Argentina.

\begin{tabular}{|c|c|c|c|}
\hline & $\begin{array}{c}\text { Normal } \\
r(\text { valor de } p)\end{array}$ & $\begin{array}{c}\text { Excesso de peso } \\
r \text { (valor de } p)\end{array}$ & $\begin{array}{c}\text { Obesidade } \\
\text { r (valor de p) }\end{array}$ \\
\hline \multicolumn{4}{|l|}{ Brasil } \\
\hline \multicolumn{4}{|l|}{ Taxas de mortalidade brutas } \\
\hline Taxa de mortalidade geral & $-0,2519(0,2049)$ & $0,2821(0,1540)$ & $0,2527(0,2035)$ \\
\hline Taxa de mortalidade por cânceres & $-0,1216(0,5456)$ & $0,1405(0,4846)$ & $0,0292(0,8852)$ \\
\hline Taxa de mortalidade por doenças cardiovasculares & $-0,0712(0,7243)$ & $0,1348(0,5025)$ & $0,1678(0,4029)$ \\
\hline \multicolumn{4}{|l|}{ Taxas de mortalidade padronizadas } \\
\hline Taxa de mortalidade geral & $-0,4454(0,0199)$ & $0,4663(0,0142)$ & $0,5330(0,0042)$ \\
\hline Taxa de mortalidade por cânceres & $-0,3797(0,0508)$ & $0,3582(0,0666)$ & $0,2706(0,1721)$ \\
\hline Taxa de mortalidade por doenças cardiovasculares & $-0,1995(0,3184)$ & $0,2585(0,1930)$ & $0,4242(0,0274)$ \\
\hline \multicolumn{4}{|l|}{ Argentina } \\
\hline Taxa de mortalidade geral & $0,5103(0,0108)$ & $-0,5752(0.0033)$ & $-0,4431(0,0301)$ \\
\hline Taxa de mortalidade por cânceres & $0,6411(0,0007)$ & $-0,5560(0,0048)$ & $-0,5725(0,0035)$ \\
\hline Taxa de mortalidade por doenças cardiovasculares & $0,5554(0,0048)$ & $-0,5647(0,0040)$ & $-0,3886(0,0606)$ \\
\hline
\end{tabular}

r: coeficiente de correlação de Pearson.

taxa de mortalidade geral e as prevalências de excesso de peso $(r=-0,58$; valor de $\mathrm{p}=0,003)$ e obesidade $(r=-0,44$; valor de $\mathrm{p}=0,030)$.

O mesmo padrão visto para a taxa de mortalidade geral foi observado nas análises para as taxas de mortalidade específicas. No Brasil, novamente os coeficientes de correlação entre o estado nutricional das capitais e as taxas de mortalidade se acentuaram após a padronização. Na Argentina, as taxas de mortalidade também se correlacionaram moderadamente com o estado nutricional das províncias, mas mais uma vez no sentido inverso do observado no Brasil (Tabela 2).

$\mathrm{Na}$ análise de regressão linear (Tabela 3) observou-se que as associações encontradas na análise bruta em ambos os países foram atenuadas após ajuste para os potenciais fatores de confusão. Cabe salientar que nas províncias argentinas a prevalência de obesidade continuou associada à taxa de mortalidade geral, mas uma relação positiva passou a ser observada após a análise ajustada. Assim, observou-se no Brasil um efeito da prevalência de obesidade sobre a taxa de mortalidade geral três vezes maior do que aquele observado na Argentina, visto que o aumento de um ponto porcentual na prevalência de obesidade acarretou um aumento médio de 0,18 morte na taxa de mortalidade geral por mil habitantes no Brasil (IC95\%: 0,01; 0,35) e de apenas 0,06 morte por mil habitantes na taxa de mortalidade geral da Argentina (IC95\%: 0,01; 0,13). No que diz respeito às mortalidades específicas, as associações encontradas na análise bruta foram atenuadas e perderam significância após o ajuste (Tabela 3).

\section{Discussão}

O presente trabalho, usando dados de pesquisas de base populacional conduzidas no Brasil e na Argentina, dois dos países mais populosos e com as maiores economias da América do Sul, mostrou que capitais brasileiras e províncias argentinas com maiores prevalências de obesidade apresentaram maior taxa de mortalidade geral, embora a medida de efeito tenha sido três vezes menor na Argentina em relação ao Brasil. Quando as taxas de mortalidade específicas foram utilizadas como desfecho em ambos os países, o efeito desapareceu após o ajuste para os fatores de confusão incluídos na análise de regressão linear.

Trabalhos conduzidos em nível individual têm mostrado que a obesidade aumenta o risco de mortalidade, especialmente de morte prematura (antes dos 70 anos), em países de alta renda 9,10,25. 
Tabela 3

Modelos de regressão linear bruto e ajustado da relação entre as prevalências do estado nutricional de 2013/2014 das capitais/províncias e as taxas de mortalidade geral, por cânceres e por doenças cardiovasculares no ano de 2015 no Brasil e na Argentina.

\begin{tabular}{|c|c|c|c|c|}
\hline \multirow[t]{2}{*}{ IMC } & \multicolumn{2}{|c|}{ Brasil } & \multicolumn{2}{|c|}{ Argentina } \\
\hline & $\beta$ bruto (IC95\%) * & $\beta$ ajustado ** (IC95\%) * & $\beta$ bruto (IC95\%) & $\beta$ ajustado *** (IC95\%) \\
\hline \multicolumn{5}{|c|}{ Taxa de mortalidade geral } \\
\hline Normal & $-0,24(-0,45 ;-0,04)$ & $-0,08(-0,23 ; 0,08)$ & $0,18(0,04 ; 0,32)$ & $-0,02(-0,10 ; 0,06)$ \\
\hline Excesso de peso & $0,22(0,02 ; 0,41)$ & $0,12(-0,02 ; 0,26)$ & $-0,21(-0,34 ;-0,08)$ & $0,01(-0,08 ; 0,09)$ \\
\hline Obesidade & $0,31(0,08 ; 0,54)$ & $0,18(0,01 ; 0,35)$ & $-0,22(-0,44 ; 0,01)$ & $0,06(0,01 ; 0,13)$ \\
\hline \multicolumn{5}{|c|}{ Taxa de mortalidade por câncer } \\
\hline Normal & $-2,08(-4,19 ; 0,04)$ & $-2,38(-4,76 ; 0,01)$ & $2,93(2,02 ; 3,83)$ & $1,07(-0,15 ; 2,28)$ \\
\hline Excesso de peso & $1,68(-0,15 ; 3,50)$ & $2,15(-0,00 ; 4,30)$ & $-2,60(-3,81 ;-1,40)$ & $-0,82(-1,96 ; 0,32)$ \\
\hline Obesidade & $1,59(-0,85 ; 4,02)$ & $2,31(-0,23 ; 4,85)$ & $-3,60(-5,96 ;-1,23)$ & $-1,10(-2,83 ; 0,63)$ \\
\hline \multicolumn{5}{|c|}{$\begin{array}{l}\text { Taxa de mortalidade por doenças } \\
\text { cardiovasculares }\end{array}$} \\
\hline Normal & $-3,19(-10,17 ; 3,78)$ & $2,37(-2,37 ; 7,11)$ & $7,23(3,77 ; 10,70)$ & $1,10(-4,17 ; 6,38)$ \\
\hline Excesso de peso & $3,54(-2,81 ; 9,88)$ & $0,06(-4,44 ; 4,57)$ & $-7,55(-11,35 ;-3,75)$ & $-2,08(-7,50 ; 3,34)$ \\
\hline Obesidade & $7,26(0,28 ; 14,25)$ & $3,94(-2,21 ; 10,10)$ & $-6,97(-13,55 ;-0,38)$ & $1,06(-3,33 ; 5,46)$ \\
\hline
\end{tabular}

* Análise realizada com as taxas padronizadas baseada na estrutura etária e sexo da população argentina;

** Ajustado para características econômicas e demográficas (IDH, escolaridade) + características comportamentais (consumo regular de frutas e hortaliças e consumo excessivo de álcool);

*** Ajustado para características econômicas e demográficas (IDH, escolaridade e média de idade) + características comportamentais (consumo regular de frutas e hortaliças e consumo excessivo de álcool).

Um estudo transversal realizado nos Estados Unidos 26 e um estudo prospectivo com homens noruegueses que estavam na faixa etária de 40 a 59 anos entre 1972 e 197527 mostraram que o excesso de peso aumenta o risco de mortalidade geral e cardiovascular em quase duas vezes, principalmente em homens. Além desses dois trabalhos, um estudo prospectivo ${ }^{11}$ mostrou que um IMC $>25 \mathrm{~kg} / \mathrm{m}^{2}$ estava associado ao aumento do risco de mortalidade, já uma meta-análise 14 concluiu que indivíduos na faixa de IMC normal apresentaram menor risco de mortalidade. Por fim, um estudo prospectivo 28 conduzido na população adulta dos Estados Unidos com dados da Pesquisa Nacional de Nutrição e Saúde (National Health and Nutrition Examination Survey - NHANES) mostrou que a obesidade tem um alto impacto na taxa de mortalidade norte-americana.

Por outro lado, uma meta-análise com 97 estudos prospectivos 8 apontou que a obesidade estaria relacionada ao aumento do risco de mortalidade, especialmente quando for igual ou maior a $35 \mathrm{~kg} / \mathrm{m}^{2}$, e que o sobrepeso (IMC entre 25 e $29,9 \mathrm{~kg} / \mathrm{m}^{2}$ ) se comportaria como um fator protetor quando comparado ao IMC normal. Os achados do presente estudo parecem suportar a primeira hipótese, ou seja, a de que o IMC elevado aumenta o risco de mortalidade, embora não tenhamos encontrado associação do excesso de peso (sobrepeso e obesidade) com as taxas de mortalidade geral e específica no nível agregado. A explicação para tal hipótese é que o excesso de tecido adiposo que caracteriza a obesidade promove distúrbios metabólicos nos diferentes sistemas do organismo 29 , expondo os indivíduos também a um maior risco de morbidade, tais como câncer 30, doença hepática 31, doença renal 32 , doenças cardiovasculares 33 e doenças respiratórias 34, levando, assim, a uma mortalidade precoce.

Cabe destacar que os resultados do presente trabalho derivam de uma análise ecológica, já os outros estudos que apontam para uma relação direta entre o IMC e o risco de mortalidade geral ou por doenças cardiovasculares são provenientes de análises individuais 13,25,26. Por outro lado, todos esses estudos de nível individual foram realizados em países de alta renda, sendo escassas as informações nacionalmente representativas para esse tipo de relação no Brasil e na Argentina, justificando, assim, a realização deste estudo ecológico. Levando-se em conta que esse tipo de delineamento é útil como gerador de novas hipóteses, certas teorias, tais como a de que o excesso de peso não estaria associado 
ao aumento do risco de mortalidade pelo papel protetor do sobrepeso e até mesmo da obesidade de grau I (IMC entre 30 e $35 \mathrm{~kg} / \mathrm{m}^{2}$ ), parecem não ser adequadas aos cenários brasileiro e argentino, visto que nossos achados em nível agregado mostraram uma associação positiva da prevalência de obesidade com a taxa de mortalidade geral em ambos os países.

Buscando uma melhor compreensão dos achados do presente estudo, nos quais, os valores de beta, como resultado da análise de regressão linear, diminuíram logo após o ajuste pelos potenciais fatores confundidores, é possível que nas províncias argentinas a idade tenha atenuado a relação do IMC com a mortalidade geral em idosos. Considerando que a idade tem sido identificada como fator de atenuação em outros estudos 25,35,36, com um efeito protetor do aumento do IMC sobre a mortalidade de pessoas maiores de 65 anos, o mesmo pode ter acontecido para as províncias argentinas. Esse possível efeito protetor do IMC em idades mais avançadas pode ocorrer devido ao fato de que pessoas acima de 60 anos, classificadas com excesso de peso e obesidade, podem ser metabolicamente saudáveis ${ }^{37}$, apresentando melhor estado nutricional e maior reserva metabólica quando comparadas àqueles idosos com menor massa corporal total 38 . Outra possível explicação poderia ser a modulação anti-inflamatória observada em idosos com doença cardiovascular e doença renal crônica 39.

Além disso, a ausência de associação entre excesso de peso e mortalidade, bem como o baixo efeito das prevalências de obesidade sobre as taxas de mortalidade no nível agregado, poderiam ser explicados também pelo aumento da prevenção secundária nos dois países, isto é, aumento na detecção precoce de comorbidades e subsequente tratamento, que deve levar à maior sobrevida 40 . Um estudo transversal 41 que comparou a mortalidade atribuível ao excesso de peso entre os anos 2005 e 2009 mostrou que, na Argentina, enquanto a prevalência de excesso de peso/obesidade aumentou rapidamente nos últimos anos, a mortalidade atribuível ao excesso de peso se manteve estável.

Por outro lado, não se pode desconsiderar a hipótese de uma provável confusão residual presente nas análises deste trabalho. Como se trata de um estudo ecológico, algum fator importante na associação entre as proporções de indivíduos classificados em cada uma das categorias de IMC das capitais/ províncias e as taxas de mortalidade pode não ter sido incluído na análise por não estar disponível nos bancos de dados utilizados. Ressalta-se que nas associações significativas que permaneceram após o ajuste para os fatores de confusão, os limites do intervalo de confiança ficaram próximos à unidade, $\mathrm{o}$ que pode evidenciar essa possível limitação.

O presente estudo também evidenciou características interessantes do Brasil e da Argentina, referentes ao IDH e ao nível de escolaridade, que merecem ser destacadas, já que alguns trabalhos ${ }^{42,43}$ têm associado tais características às prevalências de excesso de peso e obesidade. No Brasil, houve uma grande variação no IDH entre as regiões, de 0,75 nas capitais da Região Norte a 0,83 nas capitais da Região Sul, e na Argentina estas variações foram menores, de 0,82 nas províncias do Nordeste a 0,86 nas províncias das regiões Pampeana e Patagônica. Esse resultado reflete uma maior polarização entre os estados brasileiros, sendo que nas capitais do Brasil com maior IDH houve menor prevalência de obesidade, e esta relação não foi observada nas províncias argentinas. Já com relação à escolaridade, segundo o Informe Mundial de Escolaridade 44, a Argentina é o segundo país latino-americano com a menor taxa de analfabetismo, atrás apenas do Uruguai (1,9\% em 2010). Entretanto, ao analisar a proporção de indivíduos adultos com pelo menos o ensino médio completo, este estudo mostrou que a situação na Argentina (51\%) é bastante inferior àquela encontrada para a população adulta das capitais brasileiras $(65,5 \%)$.

Dentre as principais limitações deste trabalho, cabe destacar que as informações de peso e altura foram obtidas por autorrelato. Contudo, um estudo de validação mostrou elevadas correlações entre o peso, a altura e o IMC autorrelatados e medidos por antropometria ${ }^{45}$. Cabe ainda ressaltar que neste estudo utilizaram-se os dados de prevalência e, mesmo na presença de erros no autorrelato do peso e da altura, o impacto nas estimativas das medidas de prevalência será menor como consequência de menores erros na classificação nutricional. Outra limitação seria o fato de o estado nutricional das capitais/províncias ter sido avaliado única e exclusivamente pelo IMC, sem considerar outros métodos de avaliação da composição corporal, como distribuição da gordura ou outros parâmetros metabólicos. No entanto, apesar das limitações desse índice, os pontos de corte do IMC são os indicadores mais usados e recomendados para medir excesso de peso em nível populacional 46. Finalmente, a utilização de regressão linear múltipla em estudos ecológicos está sujeita ao maior efeito de multicolinearidade devido a uma maior correlação entre as variáveis no nível agregado, o que pode interferir 
na medida de efeito analisada. Tal limitação foi contornada pela análise do VIF em todos os passos da análise de regressão.

Em conclusão, este estudo mostrou que, em nível agregado, maiores prevalências de obesidade estão associadas a maiores taxas de mortalidade geral nas capitais brasileiras e províncias argentinas. Os resultados são importantes do ponto de vista clínico e de saúde pública, visto que a obesidade vem aumentando consideravelmente nos últimos anos, indicando que, no curto prazo, um maior número de indivíduos pode ter morte precoce devido a este problema.

\section{Colaboradores}

V. R. Amann contribuiu com a concepção e desenho da pesquisa, análise e interpretação dos dados, edição e aprovação final da versão a ser publicada. L. P. Santos contribuiu com a concepção e desenho do trabalho, análise e interpretação dos dados, redação e revisão crítica importante para o conteúdo intelectual. D. P. Gigante contribuiu com a concepção e desenho do trabalho, revisão crítica e aprovação final da versão a ser publicada.

\section{Referências}

1. World Health Organization. Noncommunicable diseases. http://www.who.int/news-room/ fact-sheets/detail/noncommunicable-diseases (acessado em 27/Mai/2018).

2. Instituto Brasileiro de Geografia e Estatística. Pesquisa Nacional de Saúde 2013: ciclos de vida: Brasil e grandes regiões. Rio de Janeiro: Instituto Brasileiro de Geografia e Estatística; 2015.

3. Custodio J, Elizathe L, Murawski B, Rutsztein G. Obesidad en Argentina: un desafío pendiente. Políticas de salud pública y tasas de prevalencia. Rev Mex Trastor Aliment 2015; 6:13742.

4. Cañete F, Fretes G, Sequera VG, Turnes C, Santacruz E, Paiva T, et al. Epidemiologia de la obesidad en el Paraguay. An Fac Cienc Méd (Asunción) 2016; 49:17-25.

5. Arribas-Harten C, Battistini-Urteaga T, Rodriguez-Teves MG, Bernabé-Ortiz A. Asociación entre obesidad y consumo de frutas y verduras: un estudio de base poblacional en Perú. Rev Chil Nutr 2015; 42:241-7.

6. Atalah ES. Epidemiología de la obesidad en Chile. Rev Méd Clín Condes 2012; 23:117-23.

7. Conolly A, Saunders C, Neave A. Health Survey for England 2016: adult overweight and obesity. Leeds: Health and Social Care Information Centre; 2017.

\section{Informações adicionais}

ORCID: Valeria Romina Amann (0000-0001-93168686); Leonardo Pozza dos Santos (0000-00023993-3786); Denise Petrucci Gigante (0000-00017309-5838).

8. Flegal KM, Kit BK, Orpana H, Graubard BI Association of all-cause mortality with overweight and obesity using standard body mass index categories: a systematic review and meta-analysis. JAMA 2013; 309:71-82.

9. Ponce-Garcia I, Simarro-Rueda M, CarbayoHerencia JA, Divisón-Garrote JA, ArtigaoRódenas LM, Botella-Romero F, et al. Prognostic value of obesity on both overall mortality and cardiovascular disease in the general population. PLoS One 2015; 10:e0127369.

10. Kee CC, Sumarni MG, Lim KH, Selvarajah S, Haniff J, Tee GHH, et al. Association of BMI with risk of CVD mortality and all-cause mortality. Public Health Nutr 2017; 20:1226-34.

11. Wang Z, Liu M, Pan T, Tong S. Lower mortality associated with overweight in the U.S. National Health Interview Survey: is overweight protective? Medicine (Baltimore) 2016; 95:e2424.

12. Klatsky AL, Zhang J, Udaltsova N, Li Y, Tran HN. Body mass index and mortality in a very large cohort: is it really healthier to be overweight? Perm J 2017; 21:16-142.

13. Stokes A, Preston SH. How smoking affects the proportion of deaths attributable to obesity: assessing the role of relative risks and weight distributions. BMJ Open 2016; 6:e009232. 
14. The Global BMI Mortality Collaboration. Body-mass index and all-cause mortality: individual-participant-data meta-analysis of 239 prospective studies in four continents. Lancet 2016; 388:776-86.

15. Naciones Unidas. La Agenda 2030 y los Objetivos de Desarrollo Sostenible: una oportunidad para América Latina y el Caribe. Santiago: Naciones Unidas; 2018.

16. Departamento de Vigilância de Doenças e Agravos Não Transmissíveis e Promoção da Saúde, Secretaria de Vigilância em Saúde, Ministério da Saúde. Vigitel Brasil 2014: Vigilância de Fatores de Risco e Proteção para doenças crônicas por inquérito telefônico. Brasilia: Ministério da Saúde; 2015.

17. Dirección de Promoción de la Salud y Control de Enfermedades no Transmisibles, Ministerio de Salud de la Nación; Instituto Nacional de Estadística y Censos. Tercera Encuesta Nacional de Factores de Riesgo para Enfermedades No Transmisibles. Buenos Aires: Ministerio de Salud de la Nación/Instituto Nacional de Estadística y Censos; 2015.

18. Organización Mundial de la Salud. Manual de vigilancia STEPS de la OMS: el método STEPwise de la OMS para la vigilancia de los factores de riesgo de las enfermedades crónicas. Geneva: Organización Mundial de la Salud; 2006.

19. World Health Organization. Global recommendations on physical activity for health. http://www.who.int/dietphysicalactivity/fact sheet_recommendations/en/ (acessado em 15/ Jun/2018).

20. Instituto Brasileiro de Geografia e Estatística. Projeção da população do Brasil e Unidades da Federação por sexo e idade: 2010-2060. https:// ww2.ibge.gov.br/home/estatistica/populacao/ projecao_da_populacao/2013/default.shtm (acessado em 23/Mai/2018).

21. Instituto Nacional de Estadisticas y Censos. Indicadores demográficos. https://www.indec. gob.ar/nivel4_default.asp?id_tema_1 $=2 \&$ id tema_2=24\&id_tema_3=84 (acessado em 23/ Mai/2018).

22. Centro Brasileiro de Classificação de Doenças. Classificação Estatística Internacional de Doenças e Problemas Relacionados à Saúde CID-10. São Paulo: Edusp; 2008.

23. Bussab WO, Morettin PA. Estatística básica. 5a Ed. São Paulo: Saraiva; 2003.

24. Kirkwood BR, Sterne JAC. Essential medical statistics. 2nd Ed. Malden: Blackwell Science Ltd.; 2003.

25. Wang Z, Peng Y, Liu M. Age variation in the association between obesity and mortality in adults. Obesity (Silver Spring) 2017; 25:213741.

26. Sahakyan KR, Somers VK, Rodriguez-Escudero JP, Hodge DO, Carter RE, Sochor O, et al. Normal weight central obesity, implications for total and cardiovascular mortality. Ann Intern Med 2015; 163:827-35.
27. Heir T, Erikssen J, Sandvik L. Overweight as predictor of long-term mortality among healthy, middle-aged men: a prospective cohort study. Prev Med 2011; 52:223-6.

28. Borrell LN, Samuel L. Body mass index categories and mortality risk in US adults: the effect of overweight and obesity on advancing death. Am J Public Health 2014; 104:512-9.

29. Abdelaal M, LeRoux CW, Docherty NG. Morbidity and mortality associated with obesity. Ann Transl Med 2017; 5:161.

30. Nomura DK, Long JZ, Niessen S, Hoover HS, Ng S-W, Cravatt BF. Monoacylglycerol lipase regulates a fatty acid network that promote scancer pathogenesis. Cell 2010; 140:49-61.

31. Ascha MS, Hanouneh IA, Lopez R, Tamimi TA, Zein NN. The incidence and risk factors of hepatocellular carcinoma in patients with nonalcoholic steatohepatitis. Hepatology 2010; 51:1972-8

32. Gilardini L, Zulian A, Girola A, Redaelli G, Conti A, Invitti C. Predictors of the early impairment of renal disease in human obesity. Int J Obes (Lond) 2010; 34:287-94.

33. Lakhani M, Fein S. Effects of obesity and subsequent weigh treduction on left ventricular function. Cardiol Rev 2011; 19:1-4.

34. Castro-Añón O, Pérez de Llano LA, De la Fuente Sánchez S, Golpe R, Méndez-Marote L, Castro-Castro J, et al. Obesity-hypoventilation syndrome: increased risk of death over sleep apnea syndrome. PLoS One 2015; 10:e0117808.

35. Abbate LM, Perman SM, Clambey ET, Van Pelt RE, Ginde AA. Age modifies the association between obesity and mortality in individuals hospitalized with severe sepsis. J Am Geriatr Soc 2016; 64:882-3.

36. Winter JE, MacInnis RJ, Nowson CA. The influence of age on the BMI and all-cause mortality association: a meta-analysis. J Nutr Health Aging 2017; 21:1254-8.

37. Cheng FW, Gao X, Mitchell DC, Wood C, Rolston DDK, Still CD, et al. Metabolic health status and the obesity paradox in older adults. J Nutr Gerontol Geriatr 2016; 35:161-76.

38. Oreopoulos A, Kalantar-Zadeh K, Sharma AM, Fonarow GC. The obesity paradox in the elderly: potential mechanisms and clinical implications. Clin Geriatr Med 2009; 25:643-59.

39. Doehner W, Clark A, Anker SD. The obesity paradox: weighing the benefit. Eur Heart J 2010; 31:146-8.

40. Johnson MA, Bales CW. Is there a best body mass index for older adults? Moving closer to evidence-based recommendations regarding "overweight," health, and mortality. J Nutr Gerontol Geriatr 2014; 33:1-9.

41. Acosta LD, Peláez E. Mortalidad atribuible al sobrepeso y la obesidad en Argentina: comparación entre los años 2005 y 2009. Rev Bras Estud Popul 2015; 32:277-92. 
42. Mendoza-Romo MA, Zavala-Cruz GG, Padrón-Salas A, Ortiz-Nesme FJ, Ramírez-Arriola MC, Salas-Jiménez JA. Asociación del índice de desarrollo humano y diabetes mellitus tipo 2 en unidades de medicina familiar del estado San Luis Potosí, México. Atención Familiar 2017; 24:156-9.

43. Márquez Díaz RR. Obesity: prevalence and relationship with educational level in Spain. Nutr Clín Diet Hosp 2016; 36:181-8.

44. United Nations Educational, Scientific and Cultural Organization. La Educación para Todos, 2000-2015: logros y desafíos. Informe de seguimiento de la EPT en el mundo. http://unesdoc.unesco.org/images/ 0023/002325/232565s.pdf (acessado em 23/ Mai/2018).
45. Dirección de Epidemiología, Ministerio de Salud de la Nación. Adaptación transcultural y validación de la herramienta para vigilancia de enfermedades no transmisibles en Argentina: Encuesta Nacional de Factores de Riesgo. Buenos Aires: Ministerio de Salud de la Nación; 2004.

46. Departamento de Atenção Básica, Secretaria de Atenção à Saúde, Ministério da Saúde. Estratégias para o cuidado da pessoa com doença crônica: obesidade. Brasília: Ministério da Saúde; 2014. 


\section{Abstract}

This study aimed to assess the association between prevalence of excess weight and obesity and overall and disease-specific mortality rates in Brazilian state capitals and Argentine provinces. This was an ecological study with secondary data, where the principal exposures were prevalence rates for excess weight and obesity, estimated with data from Brazil's Vigitel survey (Risk and Protective Factors Surveillance System for Chronic NonCommunicable Diseases Through Telephone Interveiew) in 2014 and Argentina's National Risk Factor Survey in 2013. Overall and specific mortality rates for the year 2015 were obtained from the Brazilian Mortality Information System in the Brazilian Health Informatics Department and the Division of Health Statistics and Information of the Argentine Ministry of Health. Brazilian mortality rates were standardized with the age structure of the Argentine population as the standard. Crude and adjusted linear regressions were used to assess the association between the prevalence rates for excess weight and obesity and the overall and specific mortality rates. After adjusting for potential confounding factors, prevalence of obesity was positively associated with the overall mortality rate both in Brazil $(\beta=0.18$; 95\% CI: 0.01; 0.35) and in Argentina $(\beta=0.06$; 95\% CI: 0.01; 0.13). There was no association with the specific cardiovascular and cancer mortality rates. We conclude that the Brazilian state capitals and Argentine provinces with the highest prevalence of obesity present higher overall mortality rates, while this association was inconsistent for the specific rates.

Body Mass Index; Mortality; Observational Study

\section{Resumen}

El objetivo de este estudio fue evaluar la asociación de la prevalencia de exceso de peso y obesidad con las tasas de mortalidad total y específica en capitales brasileñas y provincias argentinas. Estudio ecológico con datos secundarios, donde las exposiciones principales fueron las prevalencias de exceso de peso y obesidad, estimados a partir de datos de la Vigilancia de Factores de Riesgo y Protección para Enfermedades Crónicas No Transmisibles por Entrevista Telefónica (Vigitel) de 2014 (Brasil) y de la Encuesta Nacional de Factores de Riesgo de 2013 (Argentina). Las tasas de mortalidad general y específicas del año 2015 se obtuvieron del Sistema de Información sobre Mortalidad del Departamento de Informática del SUS (Brasil) y de la Dirección de Estadística e Información de Salud del Ministerio de Salud de Argentina. Las tasas de mortalidad brasileñas fueron estandarizadas considerando la estructura etaria de la población argentina como patrón. Se utilizó la regresión lineal bruta y ajustada para evaluar la asociación de las prevalencias de exceso de peso y obesidad con tasas de mortalidad general y específicas. Tras el ajuste de los potenciales factores de confusión, la prevalencia de obesidad se asoció positivamente con la tasa de mortalidad general, tanto en Brasil $(\beta=0,18$; IC95\%: 0,01; 0,35), como en Argentina $(\beta=0,06$; IC95\%: 0,01; 0,13). Para las demás tasas de mortalidad (cardiovascular y por cáncer), no hubo asociación. Se concluye que las capitales brasileñas y provincias argentinas con mayores prevalencias de obesidad presentan mayores tasas de mortalidad general, siendo esta asociación inconsistente en el caso de las tasas específicas.

Índice de Masa Corporal; Mortalidad; Estudio Observacional
Recebido em 03/Out/2018

Versão final reapresentada em 13/Mai/2019

Aprovado em 03/Jun/2019 